[4] N. Lusin, Lecons sur les Ensembles Analytiques et leurs applications, Paris 1930

[5] S. Mazurkiewicz and W. Sierpinski, Sur un problème concernañt les fonctions continues, Fund. Math. 6 (1924), pp. 161-169.

[6] R. Purves, Bimeasurable functions, Fund. Math. 58 (1966), pp. 149-157.

[7] A. H. Stone, Cardinals of closed sets, Mathematika 6 (1959), pp. 99-107.

[8] - Non-separable Borel sets, Rozprawy Mat. 28, Warszawa 1962.

[9] - On $\sigma$-disoreteness and Borel isomorphism, Amer. Journ. Math. 85 (1963), pp. $655-666$.

\title{
Non-manifold factors of Euclidean spaces
}

by

UNIVERSITY OF ROCHESTER

Rochester, New York

CARNEGIE-MELLON UNIVERSITY

Pittsburgh, Pennsylvania

A. J. Boals* (Iowa City, Ia.)

1. Introduction. The object of this paper is to define a class of factors of Euclidean $n$-space which contain some non-manifolds (i.e., Theorems 3.6 and 4.3). These factorizations will be general enough to include those given by R. H. Bing [4] and John Hemple [5].

Throughout this paper we will use the following terminology: (i) Any subset of a topological space which is homeomorphic to $I^{n}$, where $I=[0,1]$, will be called an $n$-cell. (ii) An $n$-manifold will be a paracompact Hausdorff space in which every point has a neighborhood whose closure is an $n$-cell. (iii) If $X$ is a topological space and $D \subset X$ then by int $D$ is meant the set $X-\overline{X-D}$, where $\overline{X-D}$ is the closure of $X-D$ in $X$.

\section{Separation Theorems.}

LEMMA 2.1. Let $C_{1}, C_{2}, \ldots, C_{p}$ be disjoint compact subsets of a Hausdorff space $X$. Let $D_{1}, D_{2}, \ldots, D_{p}$ be (not necessarily disjoint) $n$-cells such that for each $i=1,2, \ldots, p, C_{i} \subset \operatorname{int} D_{i}$. Then for any $[a, b] \subset E^{1}$ and $\varepsilon>0$ there exist disjoint $(n+1)$-cells $E_{1}, E_{2}, \ldots, E_{p}$ contained in $X \times(a-\varepsilon, b+\varepsilon)$ such that for each $i=1,2, \ldots, p$

(1) $C_{i} \times[a, b] \subset \operatorname{int} E_{i}$;

(2) $\Pi_{1} E_{i}=D_{i}$

where $\Pi_{1}$ is the projection of $X \times E^{1}$ onto $X$.

Proof. Let $f:[-\varepsilon, r+\varepsilon] \rightarrow[a-\varepsilon, b+\varepsilon]$ be the homeomorphism given by

$$
f(x)=\left\{\begin{array}{lll}
a+x & \text { if } & x \in[-\varepsilon, 0], \\
\left(\frac{b-a}{r}\right) x+a & \text { if } & x \in[0, r], \\
b+x-r & \text { if } & x \in[r, r+\varepsilon] .
\end{array}\right.
$$

* Submitted in partial fulfillment of the requirements for the degree of Doctor of Philosophy to Michigan State University, Department of Mathematics. The author wishes to acknowledge his indebtedness to Professor K. W. Kwon, under whose direction this thesis was written.

Supported in part by National Science Foundation grant GP-7952X. 
Let $k:[-\varepsilon, r+\varepsilon] \rightarrow[-\varepsilon, 2 p-1+\varepsilon]$ be the homeomorphism given by

$$
k(t)=\frac{2(p+\varepsilon)-1}{r+2 \varepsilon}(t+\varepsilon)-\varepsilon .
$$

For each $j=1,2, \ldots, p$ let $k_{j}$ be a homeomorphism of $[-\varepsilon, r+\varepsilon]$ onto $[-\varepsilon, 2 p-1+\varepsilon]$ with the properties:

(1) $k_{j}(-\varepsilon)=-\varepsilon$ and $k_{j}(t+\varepsilon)=(2 p-1+\varepsilon)$,

(2) $k_{j}(0)=2 j-2$,

(3) $k_{j}(r)=2 j-1$.

Let $A=\bigcup_{i} D_{i} \subset X$ and note that since $A$ is a compact Hausdorff space it is normal. Bdy $A=A-\operatorname{int} A$ and $O_{j}$ for $j=1, \ldots, p$ are closed sets. Thus there exist open sets $U_{j}$ for $j=1,2, \ldots, p$ satisfying

(1) $U_{i} \sim U_{j}=\varnothing$.if $i \neq j$

(2) $C_{i} \subset U_{i}$ for all $i=1, \ldots, p$,

(3) $U_{i} \subset \operatorname{int} A$.

By the Urysohn Lemma there exists a continuous function $g$ mapping $A$ onto $[0,1]$ such that

(1) $g\left(\bigcup_{i} O_{i}\right)=1$

(2) $g\left(A-\bigcup_{i} U_{i}\right)=0$.

Construct $h: A \times[-\varepsilon, r+\varepsilon] \rightarrow A \times[-\varepsilon, 2 p-1+\varepsilon]$ as follows:

$h(x, t)=\left\{\begin{array}{l}\left(x, g(x) k_{j}(t)+(1-g(x)) k(t)\right) \quad \text { for }(x, t) \epsilon \overline{U_{j}} \times[-\varepsilon, r+\varepsilon], \\ (x, k(t)) \text { for }(x, t) \epsilon\left(A-\bigcup_{i} U_{i}\right) \times[-\varepsilon, r+\varepsilon] .\end{array}\right.$.

For each $j=1,2, \ldots, p, h=\mathrm{id} \times k$ on $\operatorname{Bdy} \overline{U_{j}} \times[-\varepsilon, k+\varepsilon]$, hence $h$ is well-defined. $h$ is continuous since $g, k$ and $k_{j}$ are all continuous. Suppose $h\left(x_{1}, t_{1}\right)=h\left(x_{2}, t_{2}\right)$ then $x_{1}=x_{2}=x$.

First, if $x \in U_{j}$ then

or

$$
g(x) k_{j}\left(t_{1}\right)+[1-g(x)] k\left(t_{1}\right)=g(x) k_{j}\left(t_{2}\right)+[1-g(x)] k\left(t_{2}\right)
$$

$$
g(x)\left[k_{j}\left(t_{1}\right)-k_{j}\left(t_{2}\right)\right]+(1-g(x))\left[k\left(t_{1}\right)-k\left(t_{2}\right)\right]=0 .
$$

But $g(x)$ and $1-g(x) \geqslant 0$ and both $k$ and $k_{j}$ are order preserving homeo-

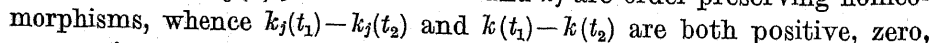
or negative together. Therefore $k\left(t_{1}\right)-k\left(t_{2}\right)=0$ and $t_{1}=t_{2}$.

Second, if $x \in A-\bigcup_{i} U_{i}$ then $k\left(t_{1}\right)=k\left(t_{2}\right)$ and again $t_{1}=t_{2}$.
Thus $h$ is one-to-one and continuous hence a homeomorphism. $h$ can be extended to a homeomorphism of $X \times[-\varepsilon, r+\varepsilon] \rightarrow X \times[-\varepsilon, 2 p-1+\varepsilon]$ by defining $h(x, t)=(x, k(t))$ on $X-A$.

For each $j=1, \ldots, p$ let $E_{j}^{\prime}$ be the $(n+1)$-cells defined by

$$
E_{j}^{\prime}=D_{j} \times\left[2 j-2-\frac{1}{4}, 2 j-1+\frac{1}{4}\right] \text {. }
$$

Now define for each $j=1,2, \ldots, p$

$$
E_{j}=(\mathrm{id} \times f)\left(h^{-1}\left(E_{j}^{\prime}\right)\right) .
$$

Clearly $E_{j} \cap E_{i}=\emptyset$ if $i \neq j$ and $\Pi_{1} E_{j}=D_{j}$. Moreover

$$
E_{i} \subset \operatorname{int} A \times(a-\varepsilon, b+\varepsilon) \text {. }
$$

If $x \in C_{i} \times[a, b]$ then

and

$$
(\text { id } \times f)^{-1}(x) \in C_{i} \times[0,2 r-1]
$$

But

$$
h \cdot(\mathrm{id} \times f)^{-1}(x) \in C_{i} \times[2 i-2,2 i-1] .
$$

whence

$$
C_{i} \times[2 i-2,2 i-1] C \operatorname{int}\left(D_{i} \times\left[2 i-2-\frac{1}{4}, 2 i-1+\frac{1}{4}\right]\right)
$$

$($ id $\times f) \cdot h^{-1} \cdot h \cdot(\operatorname{id} \times f)^{-1}(x) \epsilon(\operatorname{id} \times f) h^{-1}\left(D_{i} \times\left[2 i-2-\frac{1}{4}, 2 i-1+\frac{1}{4}\right]\right)$

and $x \in E_{i}$. Thus the $E_{i} i=1,2, \ldots, p$ satisfy all the claims of the theorem.

LEMMa 2.2. Suppose $B \neq \emptyset$ is a compact subset of int $I^{n}$ and $C$ is a compact subset of $I^{n}$ disjoint from $B$. Similarly, suppose $D \neq \varnothing$ is a compact subset of int $I^{m}$ and $E$ is a compact subset of $I^{m}$ disjoint from $D$. Then there exists an $(n+m)$-cell $G$ with the following properties:

(1) $B \times D C \operatorname{int} G C G C \operatorname{int} I^{n} \times \operatorname{int} I^{m}$,

(2) $G \cap\{(B \times E) \cup(C \times D) \cup(C \times E)\}=\varnothing$.

Proof. Let $T \subset \operatorname{int} I^{n}$ be an $n$-cell such that $T \cap(B \cup O)=\varnothing$ and $T$ is the product of its projections. Such an $n$-cell exists since $C \cap B=\emptyset$ and they are each closed. Similarly let $R \subset \operatorname{int} I^{m}$. be an $m$-cell such that $R \cap(D \cup E)=\emptyset$ and $R$ is the product of its projections. Let $\Pi_{i} T=\left[t_{i}, t_{i}^{\prime}\right]$ for each $i=1,2, \ldots, n$ and $\Pi_{j} R=\left[r_{j}, r_{j}^{\prime}\right]$ for each $j=1,2, \ldots, m$ where $\Pi_{\alpha}$ is the projection onto the ath coordinate.

Let $\delta_{1}=\min$ [distance from $B$ to $\left(B d y I^{n} \cup C\right)$, distance from $T$ to $\left.\left(B \cup C \cup \operatorname{Bdy} I^{n}\right)\right]$. Let $\delta_{2}=\min \left[\right.$ distance from $D$ to $\left(\mathrm{Bdy} I^{m} \cup E\right)$, distance from $R$ to $\left.\left(D \cup E \cup \operatorname{Bdy} I^{m}\right)\right]$. Set $\delta=\min \left(\delta_{1}, \delta_{2}\right)$. Let $k, k_{1}, k_{2}, \ldots, k_{m}$ be homeomorphisms defined as follows:

(1) $k:[0,1] \rightarrow[0,1]$ such that $k(0)=0, k(1)=1$ and $k[\delta / 2,1-\delta / 2]$ $=[1 / 4,3 / 4]$.

(2) For each $i=1,2, \ldots, m$ let $k_{i}:[0,1] \rightarrow[0,1]$ such that $k_{i}(0)=0$, $k_{i}(1)=1$ and $k_{i}\left[r_{i}, r_{i}^{\prime}\right]=[1 / 4,3 / 4]$. 
Let $U_{n}$ be an open subset of $(\delta / 2,1-\delta / 2)^{n}$ such that $B \subset U_{n}$ and $U_{n} \cap C=\emptyset$. Set $W_{n}=I^{n}-[\delta / 3,1-\delta / 3]^{n}$. By the Urysohn Lemma there exists a continuous function $g: I^{n} \rightarrow[0,1]$ such that

$$
g\left(B \cup \mathrm{Bdy} I^{n}\right)=1 \quad \text { and } g\left(I^{n}-\left(U_{n} \cup W_{n}\right)\right)=0 .
$$

Consider the following collection of maps

$$
h_{i}: I^{n} \times I^{m} \rightarrow I^{n} \times I^{m}, \quad i=1,2, \ldots, m .
$$

For $x \in I^{n}$ and $\left(y_{1}, \ldots, y_{m}\right) \in I^{m}$

$$
\left.h_{i}\left(x, y_{1}, \ldots, y_{m}\right)\right)=\left\{\begin{array}{r}
\left(x,\left(y_{1}, \ldots, y_{i-1}, g(x) k\left(y_{i}\right)+(1-g(x)) k_{i}\left(y_{i}\right), y_{i+1}, \ldots, y_{m}\right)\right) \\
\text { for }\left(x,\left(y_{1}, \ldots, y_{m}\right)\right) \epsilon \overline{U_{i}} \times I^{m} \\
\left(x,\left(y_{1}, \ldots, y_{i-1}, g(x) y_{i}+(1-g(x)) k_{i}\left(y_{i}\right), y_{i+1}, \ldots, y_{m}\right)\right) \\
\text { for }\left(x,\left(y_{1}, \ldots, y_{m}\right)\right) \epsilon \overline{W_{n}} \times I^{m} \\
\left(x,\left(y_{1}, \ldots, y_{i-1}, k_{i}\left(y_{i}\right), y_{i+1}, \ldots, y_{m}\right)\right) . \\
\text { for }\left(x,\left(y_{1}, \ldots, y_{m}\right)\right) \epsilon\left(I^{n}-\left(W_{n} \times U_{n}\right)\right) \times I^{m} .
\end{array}\right.
$$

Each $h_{i}$ is well-defined since $\overline{U_{n}} \cap \overline{W_{n}}=\emptyset, \Pi_{j} h_{j} /$ Bdy $U_{n} \times I^{m}=\Pi_{j}$ for all $j \neq i$ and

$$
\Pi_{i} h_{i} / \mathrm{Bdy} U_{n} \times I^{m}=k_{i} \Pi_{i},
$$

where again $\Pi_{i}$ is the projection onto the $i$ th coordinate axis. And $\Pi_{i} h_{i} / \mathrm{Bdy} W_{n} \times I^{m}=\Pi_{j}$ for all $j \neq i$,

$$
\Pi_{i} h_{i} / \mathrm{Bdy} W_{n} \times I^{m}=k_{i} \Pi_{i} .
$$

Clearly each $h_{i}$ is continuous and onto $I^{n} \times I^{m}$. Suppose for $x, x^{\prime} \epsilon I$ and $\left(y_{1}, \ldots, y_{m}\right),\left(z_{1}, \ldots, z_{m}\right) \in I^{m}$ we have

$$
h_{i}\left(x,\left(y_{1}, \ldots, y_{m}\right)\right)=h_{i}\left(x^{\prime},\left(z_{1}, \ldots, z_{m}\right)\right)
$$

then $x=x^{\prime}$ and $y_{j}=z_{j}$ for $\jmath \neq i$. Consider the three cases:

$$
\begin{aligned}
& \text { (1) } x \in U_{n}, \\
& \text { (2) } x \in W_{n} \text {, or } \\
& \text { (3) } x \in I^{n}-\left(U_{n} \cup W_{n}\right) \text {. }
\end{aligned}
$$

Case (1):

and

$$
g(x) k\left(y_{i}\right)+(1-g(x)) k_{i}\left(y_{i}\right)=g(x) k\left(z_{i}\right)+(1-g(x)) k_{i}\left(z_{i}\right)
$$

$$
g(x)\left(k\left(y_{i}\right)-k\left(z_{i}\right)\right)+(1-g(x))\left(k_{i}\left(y_{i}\right)-k_{i}\left(z_{i}\right)\right)=0 .
$$

Because $0 \leqslant g(x) \leqslant 1$ and $k$ as well as $k_{1}$ preserve order it follows that $y_{i}=z_{i}$.
Similar arguments show that for Cases (2) and (3), $y_{i}=z_{i}$. Thus for each $i, h_{i}$ is an injection consequently a homeomorphism.

Define $H: I^{n} \times I^{m} \rightarrow I^{n} \times I^{m}$ to be the homeomorphism $h_{1} \cdot h_{2} \cdot \ldots \cdot h_{m}$. Set

$$
J=[\delta / 2,1-\delta / 2]^{n} \times[1 / 4,3 / 4]^{m} \subset I^{n} \times I^{m} .
$$

If $(x, y) \in B \times D$ then $x \in U_{n}$ and $H(x, y) \in J$. Thus $H(B \times D) \subset J$. Let $(x, y) \epsilon(C \times(D \cup E))$ then $x \in I^{n}-U_{n}$ and there exists a $j$ such that $\Pi_{j}(y) \in I-\left[r_{j}, r_{j}^{\prime}\right]$. If $x \in W_{n}$ then $H(x, y) \notin J$. If $x \in I^{n}-\left(W_{n} \times U_{n}\right)$ then $\Pi_{j} h_{j}(x, y) \in I-[1 / 4,3 / 4]$ and $H(x, y) \notin J$. Thus $H(C \times(D \cup E)) \cap J=\emptyset$.

Note that $H / B \times I^{m}=\mathrm{id} \times k^{*}$ where $k^{*}=(K, K, \ldots, K)$ with $m$ factors. Thus it follows that $\Pi_{m}^{*} H(B \times D)$ and $\Pi_{m}^{*} H(B \times E)$ are disjoint compact subset of $[1 / 4,3 / 4]^{m} \subset I^{m}$, where $\Pi_{m}^{*}$ is the projection of $I^{n} \times I^{m}$ onto $I^{m}$. Also

$$
\Pi_{m}^{*} H(B \times D) \subset(1 / 4,3 / 4)^{m} .
$$

Let $\gamma=\min \left(\right.$ distance from $\Pi_{m}^{*} H(B \times D)$ to $\operatorname{Bdy}\left[1 / 4,3 / 4^{m}, \delta / 2\right)$. Let $U_{m}$ be an open set in $(1 / 4+\gamma / 2,3 / 4-\gamma / 2)^{m}$ such that

$$
\Pi_{m}^{*} H(B \times D) \subset U_{m} \quad \text { and } \quad U_{m} \cap \Pi_{m}^{*} H(B \times E)=\varnothing .
$$

Let $W_{m}=[1 / 4,3 / 4]^{m}-[1 / 4+\gamma / 3,3 / 4-\gamma / 3]^{m}$. There exists a continuous function $f:[1 / 4,3 / 4]^{m} \rightarrow[0,1]$ such that

(1) $f / \Pi_{m}^{*}\left(H(B \times D) \cup \operatorname{Bdy}[1 / 4,3 / 4]^{m}\right)=\mathbf{1}$,

(2) $f /[1 / 4,3 / 4]^{m}-\left(U_{m} \cup W_{m}\right)=0$.

Let $\psi, \psi_{1}, \ldots, \psi_{n}$ be homeomorphisms defined as follows:

(1) $\psi:[\delta / 2,1-\delta / 2] \rightarrow[\delta / 2,1-\delta / 2]$ such that $\psi(\delta / 2)=\delta / 2, \psi(1-\delta / 2)$ $=1-\delta / 2$ and $\psi[\delta / 2+\gamma / 2,1-\delta / 2-\gamma / 2]=[1 / 4,3 / 4]$.

(2) For each $i=1,2, \ldots, n$ let $\psi_{i}:[\delta / 2,1-\delta / 2] \rightarrow[\delta / 2,1-\delta / 2]$ such that $\psi_{i}(\delta / 2)=\delta / 2, \psi_{i}(1-\delta / 2)=1-\delta / 2$ and $\psi_{i}\left[t_{i}, t_{i}^{\prime}\right]=[1 / 4,3 / 4]$. Consider the following collection of maps:

$$
\theta_{i}: I^{n} \times I^{m} \rightarrow I^{n} \times I^{m} \quad i=1,2, \ldots, n .
$$

For $\left(x_{1}, x_{2}, \ldots, x_{n}\right) \in I^{n}$ and $y \in I^{m}$

$$
\theta_{i}\left(\left(x_{1}, \ldots, x_{n}\right), y\right)=\left\{\begin{array}{c}
\text { id } \quad \text { for }\left(\left(x_{1}, \ldots, x_{n}\right), y\right) \in\left(I^{n} \times I^{m}\right)-\operatorname{int} J, \\
\left(\left(x_{1}, \ldots, x_{i-1}, f(y) \psi\left(x_{i}\right)+(1-f(y)) \psi_{i}\left(x_{i}\right), x_{i+1}, \ldots, x_{n}\right), y\right) \\
\text { for }\left(\left(x_{1}, \ldots, x_{n}\right), y\right) \epsilon[\delta / 2,1-\delta / 2]^{n} \times U_{m}, \\
\left(\left(x_{1}, \ldots, x_{i-1}, f(y) x_{i}+1-f(y) \psi_{i}\left(x_{i}\right), x_{i+1}, \ldots, x_{n}\right), y\right) \\
\text { for }\left(\left(x_{1}, \ldots, x_{n}\right), y\right) \epsilon[\delta / 2,1-\delta / 2]^{n} \times W_{m}, \\
\left(\left(x_{1}, \ldots, x_{i-1}, \psi_{i}\left(x_{i}\right), x_{i+1}, \ldots, x_{n}\right), y\right) \\
\text { on }[\delta / 2,1-\delta / 2]^{n} \times\left(\left[1 / 4,3 / 4^{m}\right]-\left(U_{m} \cup W_{m}\right)\right) .
\end{array}\right.
$$


Each $\theta_{i}$ is well-defined since $\overline{W_{m}} \cap \overline{U_{m}}=\varnothing$. By an argument exactly luke the one given above for $h_{i}$, each $\theta_{i}$ is a homeomorphism. Define $\theta=\theta_{1}$ 。 $\circ \theta_{2} \circ \ldots \circ \theta_{n}$

Set

$$
J^{\prime}=[1 / 4,3 / 4]^{n} \times[1 / 4+\gamma / 2,3 / 4-\gamma / 2]^{m} \subset J .
$$

If $(x, y) \in B \times D$ then $\theta H(x, y) \in J^{\prime}$. Thus $\theta H(B \times D) \subset J^{\prime}$. If $(x, y)$ $\epsilon((C \times D) \cup(C \times E))$ then $H(x, y) \notin J$ hence $\theta \cdot H(x, y) \notin J^{\prime}$. Suppose $(x, y) \in B \times E$ then $\Pi_{m}^{*} H(x, y) \in[1 / 4,3 / 4]^{m}-U_{m}$ and there exists a $j$ such that $\Pi_{j}(x) \notin\left[t_{j}, t_{j}^{\prime}\right]$. If $\Pi_{m}^{*} H(x, y) \in W_{m}$ then $\theta \cdot H(x, y) \notin J^{\prime}$ since

$$
\Pi_{m}^{*} \theta \cdot H(x, y)=\Pi_{m}^{*} H(x, y) \quad \text { and } \quad W_{m} \cap \Pi_{m}^{*} J^{\prime}=\emptyset .
$$

If $\Pi_{m}^{*} H(x, y) \in \Pi_{m}^{*}(J)-W_{m}-U_{m}$ then $\Pi_{j} \Pi_{m}^{*} H(x, y) \notin[1 / 4,3 / 4]$ and $\theta \cdot H(x, y) \notin J^{\prime}$. Therefore $\theta \cdot H\{(B \times E) \cup(C \times D) \cup(C \times E)\}$ is contained in $I^{n} \times I^{m}-J^{\prime}$

Define $G=H^{-1} \cdot \theta^{-1}\left(J^{\prime}\right) . G$ is the $(n+m)$-cell contained in $\operatorname{int} I^{n} \times$ $\times \operatorname{int} I^{m}$ satisfying properties 1 and 2 of theorem.

Note that $J^{\prime}$ defined in the above proof in the product of cells. Thus a proof similar to that of Theorem 2.1 would prove the following theorem.

Limara 2.3. Suppose $B_{i}, i=1,2, \ldots, p$ are disjoint compact subsets of int $I^{n}$, one of which is non-empty, and $C$ is a compact subset of $I^{n}$ disjoint from $B=\bigcup_{i} B_{i}$. Similarly suppose $D_{j}, j=1,2, \ldots, q$ are disjoint compact subsets of int $I^{m}$, one of which is non-empty, and $E$ is a compact subset of $I^{m}$ disjoint from $\bigcup_{i} D_{i}=D$. Then there exist $(n+m)$-cells $G_{i j} i=1,2, \ldots, p$, $j=1,2, \ldots, q$ such that
(1) $G_{i j} \cap G_{r s}=\emptyset$ if $i \neq r$ or $j \neq s$,
(2) $B_{i} \times D_{j} \subset \operatorname{int} G_{i j} \subset G_{i j} \subset \operatorname{int}\left(I^{n} \times I^{m}\right)$,
(3) $\bigcup_{i} \bigcup_{j} G_{i j} \cap\{(C \times D) \cup(B \times \dot{E}) \cup(C \times E)\}=\emptyset$.

3. A class of factorizations of $E^{n}$. In this section we shall define a class of upper semi-continuous decompositions of $E^{n}$ and prove that the associated decomposition spaces are factors of $E^{n+1}$. This class contains the decompositions for each of the spaces (a) "dogbone space", (b) "unused example" and (c) "segment space" ([5]).

Definition 3.1. Suppose $\alpha$ is an arc in $E^{n}$ (i.e. $\alpha=h[0,1]$ for some homeomorphism $\left.h: I \rightarrow E^{n}\right)$ such that $P=\Pi_{1} / \alpha$ is an injection, where said to have property $E^{n}$ onto the 1 st coordinate. In this case $\alpha$ will be
Let $\alpha$ be an arc with property QS and assume that $\Pi_{1} h(1)=b$ and $\Pi_{1} h(0)=a$ with $a<b$. Define the continuous function $f: E^{1} \rightarrow E^{n}$ by

$$
f(t)=\left\{\begin{array}{lll}
P^{-1}(a) & \text { for } & t \leqslant a \\
P^{-1}(t) & \text { for } & a \leqslant t \leqslant b \\
P^{-1}(b) & \text { for } & b \leqslant t .
\end{array}\right.
$$

Define the homeomorphism $k: E^{1} \times E^{n-1} \rightarrow E^{1} \times E^{n-1}$ by $k(t, x)$ $=(t, x-f(t))$. For any $\varepsilon>0$ let

$$
\begin{aligned}
& C_{1}=\left\{z \mid z \in E^{n},\|z-a\| \leqslant \varepsilon\right\}, \\
& C_{2}=\left\{z \mid z \in E^{n},\|z-b\| \leqslant \varepsilon\right\}, \\
& C_{3}=\left\{z \mid z \in E^{n}, a \leqslant \Pi_{1} z \leqslant b \text { and }\left\|z-\Pi_{1} z\right\| \leqslant \varepsilon\right\}
\end{aligned}
$$

then $Q_{8}=C_{1} \cup C_{2} \cup C_{3}$ is an $n$-cell containing $\Pi_{1}(\alpha)$. The $n$-cell $k^{-1}\left(Q_{\varepsilon}\right)$ will be called an $\varepsilon$-radial neighborhood of $\alpha$.

Remark 3.1. Note that if $\alpha$ is an arc with property QS then for any $\varepsilon>0$ the $\varepsilon$-radial neighborhood of $\alpha$ intersects the planes $\Pi_{1}^{-1}(t)$ $=R_{t}=\left\{(t, y) !^{\prime}(t, y) \epsilon t \times \mathbb{E}^{n-1}\right\}$ is void, a single point, or an $(n-1)$ - cell.

Remark 3.2. Suppose $\alpha$ is an arc which has property QS. Since the homeomorphism used to define radial neighborhood is uniformly continuous, it follows that for any $\varepsilon>0$ there exists a $\delta>0$ and a collection of planes $R_{i}=\Pi^{-1}\left(t_{i}\right)$ with $t_{1}=u<t_{2}<\ldots<t_{p}=b$ such that the $R_{i}$ cut the $\delta$-radial neighborhood of $a$ into $(p+1) n$-cells $C_{i} ; i=0,1, \ldots, p$ and $\operatorname{diam} C_{i} \leqslant \varepsilon$.

Let $A_{1}, A_{2}, \ldots$ be a sequence of compact $n$-manifolds (not necessarily connected) in $E^{n}$ satisfying

P1. $A_{i+1} \subset \operatorname{int} A_{i}$ for all $i=1,2,3, \ldots$

P2. Each component of $A_{\infty}=\bigcap_{i} A_{i}$ is an arc with property QS. Let $\Gamma_{n}^{\prime}$ be the class of upper semi-continuous decompositions of $E^{n}$ into $\operatorname{arcs} A_{\infty}$ and points of $E^{n}-A_{\infty}$. Further let $\Gamma_{n}$ be the class of associated decomposition spaces.

LEMMA 3.1. Suppose $\varepsilon>0$ and $A_{i}$ are as defined above, then there exists a finite collection of $n$-cells $U_{i}$ satisfying:

1. For each $U_{i}$ there exists an arc $\alpha_{1} \subset A_{\infty} \cap$ int $U_{i}$ such that the distance from $x$ to $\mathrm{Bdy} U_{i}$ is less than $\varepsilon$ for all $x \in a_{i}$.

2. There exists an integer $m$ such that if $A$ is a component of $A_{m}$ then $A \subset \operatorname{int} U_{i}$ for some $i$.

Proof. For each arc $\alpha \epsilon A_{\infty}$ let $N_{a}$ be the $\varepsilon / 2$-radial neighborhood of $\alpha$. For each $N_{a}$ there exists a neighborhood $V_{a} \subset N_{a}$ with the property that if an arc $\beta \subset A_{\infty}$ intersects $V_{\alpha}$ non-trivially then $\beta \subset N_{\alpha}$. The existence Fundamenta Mathematicae, T. LXVIII 
of such $V_{a}$ 's follows from the fact that the decomposition of $E^{n}$ into th ares of $A_{\infty}$ and the points of $E^{n}-A_{\infty}$ is an upper semi-continuous decomposition. The collection of sets $\left\{V_{a} \mid \alpha \subset A_{\infty}\right\}$ is an open cover of the compact set $A_{\infty}$. Thus there is a finite subcollection $V_{1}, V_{2}, \ldots, V_{p}$ which covers $A_{\infty}$. Let $N_{1}, N_{2}, \ldots, N_{p}$ be the corresponding $N_{a}$ 's. Note that by the choice of the $V_{\alpha}$ 's we have each arc $\alpha \subset A_{\infty}$ contained in the interior of at least one $N_{i}$. For each arc $\alpha \subset A_{\infty}$ there exists an integer $m(\alpha)$ such that

1. $\alpha \subset A_{m(\alpha)}^{a} \subset A_{m(\alpha)}$ where $A_{m(\alpha)}^{\alpha}$ is the component of $A_{m(\alpha)}$ containing $\alpha$;

2. $A_{m(a)}^{a} \subset \operatorname{int} N_{i}$, for some $i=1,2, \ldots, p$.

The collection $\left\{\operatorname{int} A_{m(a)}^{\alpha} \mid \alpha \subset A_{\infty}\right\}$ is an open cover of $A_{\infty}$. Therefore there is a finite subcover. From this collection of $\boldsymbol{A}_{m(a)}^{a}$ 's there is one with largest subscript $m(\alpha) . m=m(\alpha)$ is the desired integer. Each $N_{i}$ is the $\varepsilon / 2$-radial neighborhood of some $\alpha \subset A_{\infty}$. Therefore the collection $U_{i}=N_{i}$ satisfies the claims of the Lemma.

Lemma 3.2. Suppose $A_{i}, i=1,2, \ldots$, are defined as above and $A$ is a component of $A_{r}$ for some $r$. Given $\varepsilon>0$ then there exist integers $\gamma(1)$, $\gamma(2), \ldots, \gamma(m+1)$ and sets $K_{i j} \subset A \times \mathbb{E}^{1}, i=1,2, \ldots, s ; j=1,2, \ldots, m$ which satisfy the following conditions:

1. For each $i, K_{i_{0}}$ is an $(n+1)$-cell and $K_{i j}$ is the disjoint union of $(n+1)$ - cells $K_{i j k}, k=1,2, \ldots, \mu(i, j)$;

2. $K_{i 0} \cap K_{e 0}=\varnothing$ if $i \neq e$;

3. $\bigcup_{i} K_{i j} \subset\left(A_{\gamma(j)} \cap A\right) \times[j, 2 m+1-j], \bigcup_{i} K_{i j+1} \subset\left(\operatorname{int} A_{\gamma(j)} \cap A\right) \times$ $\times(j, 2 m+1-j)$ for each $j$;

4. For each $i K_{i 0}$ can be written as the union of $(n+1)$-cells $D_{i e}$, $e=0,1, \ldots, m$, such that $D_{i e} \cap D_{i v}=\operatorname{Bdy} D_{i e} \cap \operatorname{Bdy} D_{i v}$ is an $n-c e l l$ if $|e-v|=1$ and is void if $|e-v|>1$;

5. Diameter of $\Pi_{n}^{*}\left(D_{i e}\right)<\varepsilon$ for all $i$, e, where $\Pi_{n}^{*}$ is the projection $E^{n} \times E^{1} \rightarrow E^{n}$;

6. $D_{i e} \cap D_{i v} \cap K_{i j k}$ is either void or an $n$-cell.

Proof. Let the $\varepsilon$ of Lemma 3.1 be the min ( $\varepsilon$, distance from $A_{\infty} \cap A$ to $\mathrm{Bdy} A$ ) hence there exists a finite set of $n$-cells $K_{i 0}^{\prime}, i=1,2, \ldots, s$ and an integer $\gamma(1)$ satisfying:

a. $K_{i 0}^{\prime} \subset \operatorname{int} A$ for all $i$;

b. If $A^{\prime}$ is a component of $A_{\gamma(1)} \cap A$ then $A^{\prime} \subset \operatorname{int} K_{i 0}^{\prime}$ for some $i$.

Note that the $K_{i 0}^{\prime}$ may not be disjoint. By Remark 2.2 each $n$-cell $K_{i 0}^{\prime}$ can be chosen so that there is a finite set of planes $R_{i j}, j=1,2, \ldots, m_{i}$ which cut $K_{i 0}^{\prime}$ into $\left(m_{i}+1\right) n$-cells $D_{i j}^{\prime}$ such that

$$
D_{i j}^{\prime} \cap D_{i v}^{\prime}=\operatorname{Bdy} D_{i j}^{\prime} \cap \operatorname{Bdy} D_{i v}^{\prime}
$$

is an (n-1)-cell if $|j-v|=1$ and is void if $|j-v|>1$. Without loss of generality assume $m_{i}=m$ for all $i$.

Similarly apply Lemma 3.1 to each component of $A_{\gamma(1)} \cap A$ to obtain an integer $\gamma(2)$ and sets $K_{i 1}^{\prime}$ where $K_{i 1}^{\prime}$ is the union of $n$-cells $K_{i 1 k}^{\prime}$, $k=1,2, \ldots, \mu(i, 1)$, satisfying:

(i) If $A^{*}$ is a component of $A_{\gamma(2)} \cap A$ then

$$
A^{*} \subset \operatorname{int} K_{i 1 k}^{\prime} \subset K_{i 1 k} \subset \operatorname{int} A^{\prime} \subset K_{i 0}^{\prime}
$$

for some $k$ and some component $A^{\prime}$ of $A_{\gamma(1)} \cap A$.

(ii) $K_{i 1 k} \not \subset R_{i j}$ is either void or an $(n-1)$-cell.

Condition (ii) actually follows from the proof of Lemma 3.1. Continue this procedure to obtain the integers $\gamma(3), \gamma(4), \ldots, \gamma(m+1)$ and sets $K_{i j}^{\prime}$ as well as $n$-cells $K_{i j k}^{\prime}$ satisfying conditions analogous to (i) and (ii).

For each $i$ and $j$ define $W_{i j l}$ to be the union of the components of $A_{\gamma(j+1)} \cap A$ which are contained in $K_{i j l}^{\prime}$ but not in $K_{i j p}^{\prime}$ for any $p<l$. Note that $W_{i j l}$ are compact and $W_{i j k} \cap W_{i j l}=\varnothing$ if $k \neq l$. Let $\left\{W_{i 01}\right\}$ and $\left\{K_{i 0}^{\prime}\right\}$ be respectively $\left\{C_{i}\right\}$ and $\left\{D_{i}\right\}$ of Lemma 2.1 and let $a-\varepsilon=0$ and $b+\varepsilon=2 m+1$. Then define $K_{i 0}=E_{i}$ of Lemma 2.1. By the proof of Lemma 2.1 we see that $K_{i 0}$ can be written as the union of $(n+1)$-cells $D_{i l}$ such that $\Pi_{n}^{*} D_{i l}=D_{i l}^{\prime}$. Further the $D_{i l}$ satisfy condition 4 .

In general let $\left\{W_{i j k}\right\}$ and $\left\{K_{i j k}^{\prime}\right\}$ be respectively $\left\{C_{i k}\right\}$ and $\left\{D_{i k}\right\}$ of Lemma 2.1 and let $\varepsilon=1 / 2, a=j$ and $b=2 m+1-j$. If $K_{i j k}=E_{i k}$ of Lemma 2.1 and $K_{i j}=\bigcup_{k} E_{i k}$ then conditions 1 through 5 are clearly satisfied and condition 6 follows from (ii) above.

Remark 3.3. Note that if $i \neq r$ and $A^{\prime}$ is a component of $A_{\gamma(i+1)} \cap A$ contained in $K_{r j}^{\prime}$ then $K_{i j+1} \cap A^{\prime} \times E^{1}=\emptyset$ since $K_{i j}^{\prime} \cap K_{r j}^{\prime} \subset A-A_{y(j+1)}$. Also $K_{i j p}^{\prime} \cap K_{i j p}^{\prime}=\varnothing$ if they are not in the same $n$-cell of $K_{i j-1}^{\prime}$.

The proof of the next lemma is based on the following known result.

THEOREM. Suppose that $A$ is an n-cell which is the union of two n-cells $A_{1}$ and $A_{2}$ with the properties that $A_{1} \cap A_{2}$ and $\mathrm{Bdy} A_{1} \cap \mathrm{Bdy} A_{2}$ are $(n+1)$-cells and $A_{1} \cap A_{2} \subset \operatorname{Bdy} A_{1} \cap \operatorname{Bdy} A_{2}$. If $B \subset A, B$ is compact and $B \cap \operatorname{Bdy} A \subset A_{2}$ then there exists a homeomorphism $h$ of $A$ onto $A$ which is fixed on the $\mathrm{Bdy} A$ and such that $h(B) \subset A_{\mathbf{2}}$.

LEMMA 3.3. For $\varepsilon \geqslant 0$ and $A$ a component of $A_{r}$ (where $A_{i}, i=1,2, \ldots$, are defined as above) let $\gamma(f), D_{i l}, K_{i j}$, and $K_{i j k}$ be as in Lemma 3.2. Then there exists a homeomorphism $h: E^{n} \times E^{1} \rightarrow E^{n} \times E^{1}$ such that the following hold:

$$
\begin{aligned}
& \text { 1. } h=\mathrm{id} \text { on complement of } \bigcup_{i} K_{i 1} \text {; } \\
& \text { 2. } h=\mathrm{id} \text { on the complement of }
\end{aligned}
$$

$\bigcup_{i}\left(\left(K_{i 1} \cap\left(D_{i 0} \cup D_{i 1}\right)\right) \cup\left(K_{i 2} \cap\left(D_{i 1} \cap D_{i 2}\right)\right) \cup \ldots \cup\left(K_{i m} \cap\left(D_{i m} \cup D_{i m}\right)\right)\right) ;$ 


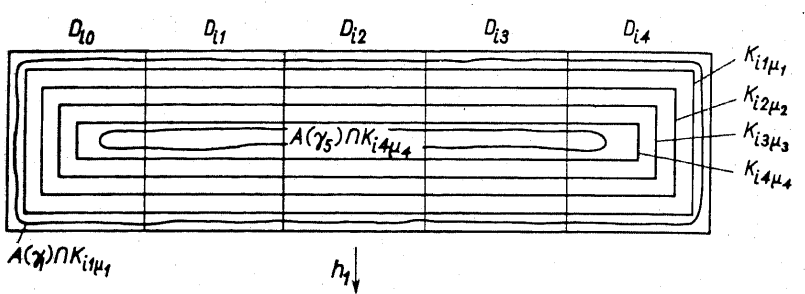

$$
\begin{aligned}
& \text { 3. If } A^{\prime} \subset A_{\gamma(j+1)} \cap A \text { and } K_{i j k} \supset A^{\prime} \times[j, 2 m+1-j] \text { then } \\
& h\left(\left(D_{i 0} \cup \ldots \cup D_{i j}\right) \cap A^{\prime} \times([j, j+1] \cup[2 m-j, 2 m+1-j])\right)
\end{aligned}
$$

is contained in $D_{i \psi} \cup D_{i \varphi+1}$ where $\psi=\min \left(j, \max \{\theta\} \quad K_{i e k} \cap D_{i e} \neq \emptyset\right.$, $\left.\left.K_{i e k} \supset A^{\prime}\right\}\right)$.

Before reading the proof of Lemma 3.3 it may be helpful to look at Figures 1 and 2. The homeomorphism $h$ will be obtained as the composition of homeomorphisms $h_{m-1} \circ h_{m-2} \circ \ldots \circ h_{1}$. Figure 1 illustrates how the $h_{j}$ will be constructed. The shaded region of Figure 2 is that part of $\boldsymbol{A} \times$ $\times[0,2 m+1]$ which is not moved by $h$.

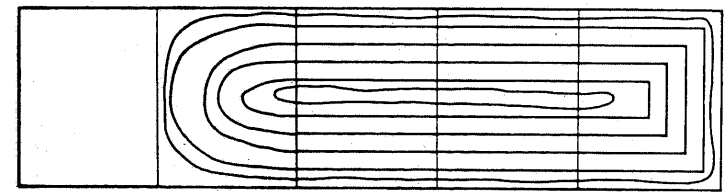

$h_{2} \downarrow$

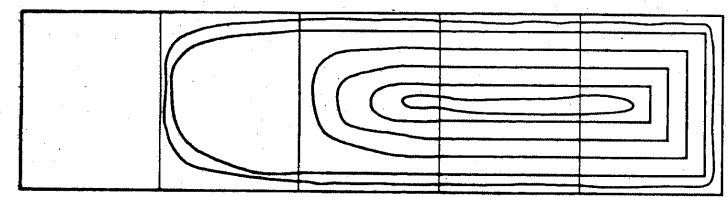

$n_{3}$

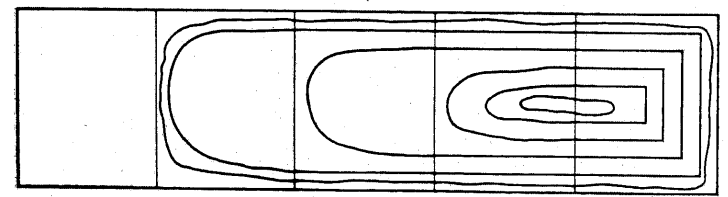

$h_{4} \downarrow$

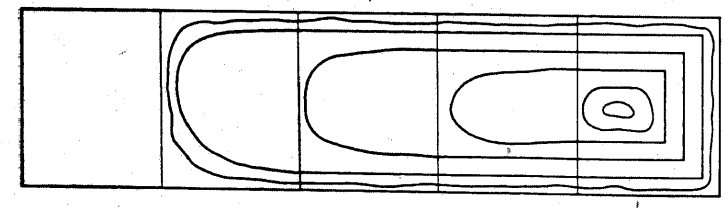

Fig. i

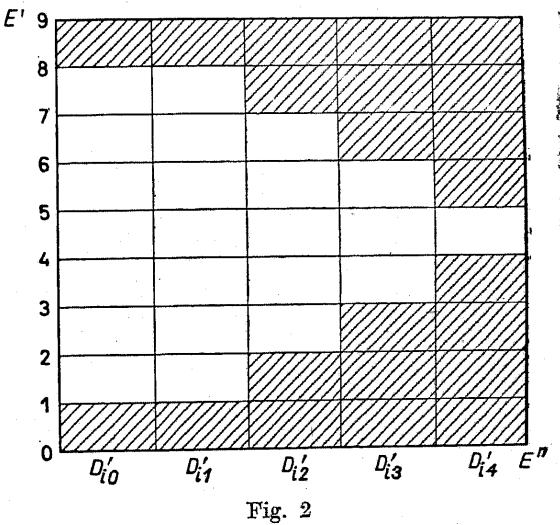

Proof of Lemma 3.3. Let $h_{1}: E^{n} \times E^{1} \rightarrow E^{n} \times E^{1}$ be a homeomorphism defined as follows:

$$
h_{1}=\text { id } \quad \text { on } \cdot E^{n} \times E^{\prime}-\bigcup_{i}\left(K_{i 1} \cap\left(D_{i 0} \cup D_{i 1}\right)\right) .
$$

For each $i$ and $A^{\prime}$ a component of $A_{\gamma(2)}$ with $A^{\prime} \times[1,2 m] \subset K_{i 1 k}$, then a. If $K_{i 1 k} \cap D_{i 0}=\varnothing$ or $K_{i 1 k} \cap D_{i 1}=\emptyset$ then

$$
h_{1}=\mathrm{id} \text { on } K_{i 1 k} \text {; }
$$

b. If $K_{i 1 k} \cap K_{i 0} \cap D_{i 1} \neq \varnothing$ then

$h_{1}=\mathrm{id}$ on $\mathrm{Bdy} K_{i 1} \cap\left(D_{i 0} \cup D_{i 1}\right)$ and $h_{1}\left(A^{\prime} \times[1,2 m] \cap\left(D_{i 0} \cup D_{i 1}\right)\right) \subset D_{i 1}$.

$h_{1}$ as defined exists since $A^{\prime} \times[1,2 m]$ is compact, $K_{i 1 k} \cap\left(D_{i 0} \cup D_{i 1}\right)$ is the un common boundary and $\left(A^{\prime} \times[1,2 m]\right) \cap \operatorname{Bdy}\left(K_{i j k} \cap\left(D_{i 0} \cup D_{i 1}\right)\right) q \subset D_{i 1}$ and $K_{i j k} \cap K_{i j l}=\varnothing$ if $k \neq l$. 
Now proceed inductively to define $h_{j}$ for $j=2,3, \ldots, m-1$. As a notational aid define $L_{i j}=\left(D_{i 0} \cup D_{i 1} \cup \ldots \cup D_{i j}\right) \cap K_{i j}$.

Define $h_{j}: E^{n} \times E^{\prime} \rightarrow E^{n} \times E^{\prime}$ as follows:

a. $h_{j}=\operatorname{id~on~} E^{n} \times E^{1}-\left(h_{j-1} \circ h_{j-2} \circ \ldots \circ h_{1}\left(\bigcup_{i} L_{i j}\right)\right)$.

For each $i$ and $A^{\prime}$ a component of $A_{\gamma(j+1)} \cap A$ with $A^{\prime} \times[j, 2 m+1-j]$ $\subset K_{i j k}$ then

b. If $H \cap D_{i j-1}=\varnothing$ or $H \cap D_{i j}=\varnothing$ then

$$
h_{j}=\mathrm{id} \text { on } H=\left(h_{j-1} \circ h_{j-2} \circ \ldots \circ h_{1}\left(K_{i j k}\right)\right) \text {; }
$$

c. If $H \cap D_{i j-1} \cap D_{i j} \neq \emptyset$ then let $h_{j}$ be such that

$$
h_{j}\left(h_{i-1} \circ h_{j-2} \circ \ldots \circ h_{1}\left(A^{\prime} \times[j, 2 m+1-j] \cap\left(D_{i j-1} \cup D_{i j}\right)\right)\right)
$$

is contained in $D_{i j}$.

$h_{j}$ exists since $A^{\prime} \times[j, 2 m+1-j]$ is compact,

$$
h_{j-1} \circ \ldots \circ h_{1}\left(K_{i j k} \cap\left(D_{i j-1} \cup D_{i j}\right)\right)
$$

is the union of two $(n+1)$-cells which intersect in an $n$-cell in their common boundary and

$$
\left.h_{j-1} \circ \ldots \circ h_{1}\left(A^{\prime} \times[j, 2 m+1-j]\right) \frown \mathrm{Bdy} h_{j-1} \circ \ldots \circ h_{1}\left(K_{i j k}\right) \cap\left(D_{i j-1} \cup D_{i j}\right)\right)
$$

is contained in $D_{i j}$.

Set $h=h_{m-1} \circ h_{m-2} \circ \ldots \circ h_{1}$. Clearly conditions 1 and 2 are satisfied by $h$. To see that condition 3 is satisfied let

$$
x \in\left(A_{\gamma(j+1)} \cap A\right) \times([j, j+1] \cup[2 m-j, 2 m+1-j]) .
$$

There exists some component $A^{\prime} \subset A_{\gamma(j+1)} \cap A$ such that

$$
x \in A^{\prime} \times([j, j+1] \cup[2 m-j, 2 m+1-j])
$$

and a unique $k_{i j k}$ containing $x$. Let $\psi=\min \left(j, \max \left\{e \mid K_{i e k} \cap D_{i e} \neq \emptyset\right.\right.$, $\left.\left.D_{i e k} \supset A^{\prime}\right\}\right)$.

Case 1. If $\psi<j$ then

$$
h(x)=h_{m-1} \circ \ldots \circ h_{\psi} \circ \ldots \circ h_{1}(x)=h_{\psi} \circ \ldots \circ h_{1}(x) \subset D_{i \psi} \cup D_{i \psi+1} .
$$

Case 2. If $\psi=j$ then

$$
h(x)=h_{m-1} \circ \ldots \circ h_{j+1} \circ h_{j} \circ \ldots \circ h_{1}(x)=h_{j+1} \circ h_{j} \circ \ldots \circ h_{1}(x)
$$

which is a point in $D_{i j} \cup D_{i j+1}$.

LEMma 3.4. Suppose $\varepsilon>0$ and $A$ is a component of $A_{r}$ (where $A_{i}$ $i=1,2, \ldots$ are defined as above). Then there exists an integer $N$ and $a$ uniformly continuous homeomorphism $h: E^{n} \times E^{1} \rightarrow E^{n} \times E^{1}$ which is the identity on $E^{n+1}-\left(A \times E^{1}\right)$ and such that for each $w \in \mathbb{B}^{1}$
(1) $\Pi_{n+1}(h(A \times w)) \subset[w-2 m-1, w+2 m+1]$,

(2) $\operatorname{diam}\left(\Pi_{n}^{*}\left(A^{\prime} \times \dot{w}\right)\right)<4 \varepsilon$

where $A^{\prime}$ is a component of $A_{n} \cap A, \Pi_{n+1}$ is the projection of $E^{n} \times E^{1}$ onto $E^{1}$, and $I_{n}^{*}$ is the projection onto $E^{n}$.

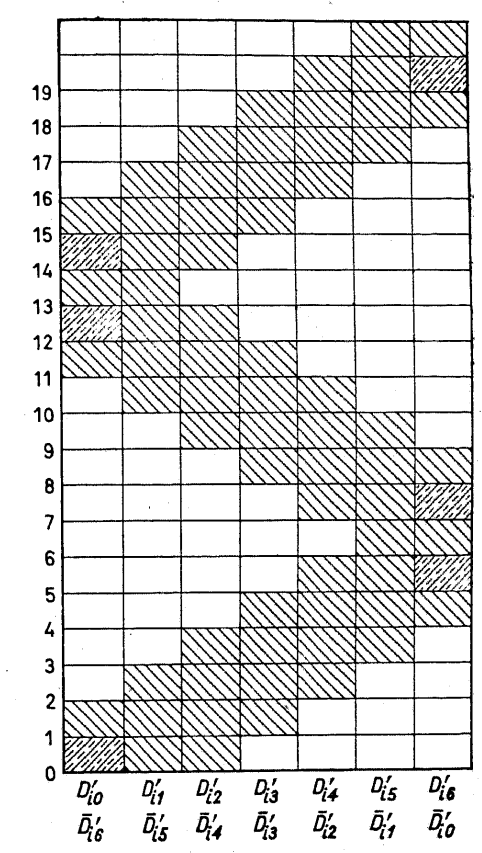

Fig. 3

Figure 3 shows how to apply Lemma 3.3 to prove Lemma 3.4. In Figure 3 only one sequence $K_{i 1 k}, K_{i 2 k}, \ldots, K_{i m+1 k}$ containing a component of $A_{N} \cap A$, is shown. The $(n+1)$ - cells in the figure are shown as if they intersect each of the $(n+1)$-cells $D_{i 0}, D_{i 1}, \ldots, D_{i m}$. This may not be the case; however, an analogous figure is obvious.

Proof. Apply Lemma 2.3 to $A \times E^{1}$ and integers $m$ and $\gamma(m+1)$ and sets $D_{i l}, K_{i j}$ and $K_{i j k}$. Set $\dot{N}=\gamma(m+1)$ for $g=0, \pm 1, \pm 2, \ldots$ let

$$
\begin{array}{ll}
x_{g}=g(2 m+2), & x_{g}^{\prime}=x_{g}+m+1, \\
y_{g}=g(2 m+2)+2 m+1, & y_{g}^{\prime}=y_{g}+m+1 .
\end{array}
$$


Note that $D_{i l}, K_{i j}$, and $K_{i j k} \subset A \times\left[x_{0}, y_{0}\right]$ by suitable translations of $E^{\prime}$ we get sets analogous to $D_{i l}, K_{i l}$, and $K_{i j k}$ in $A \times\left[x_{g}, y_{g}\right]$ for each $g$. Apply Lemma 3.3 to $A \times\left[x_{g}, y_{g}\right]$ for each $g$. Define $\overline{D_{j l}}=D_{i m-l}$ and apply Lemma 3.3 to $A \times\left[x_{l}^{\prime}, y_{l}^{\prime}\right]$ using $\overline{D_{i l}}$ in place of $D_{i l}$. Thus there exists a homeomorphism which is uniformly continuous. By the choice of $x_{g}, y_{g}, x_{g}^{\prime}$ and $y_{g}^{\prime}$ and Remark 3.3 there exist integers $i$ and $k$ such that

$$
\Pi_{n}^{*} h\left(A^{\prime} \times w\right) \subset \Pi_{n}^{*}\left(D_{i k} \cup D_{i k+1} \cup \bar{D}_{i m-k-2} \cup \bar{D}_{i m-k-3}\right)
$$

for each component $A^{\prime} \subset A \cap A_{N}$ and $w \in E^{1}$. Note that $i$ and $k$ depend on $A^{\prime}$ and $w$. Diameter $\Pi_{n}^{*}\left(D_{i l}\right)<\varepsilon$ for all $i$ and $l$. Thus condition (2) is satisfied. For $w \in E^{1}$ there exist $x_{g}, y_{g}, x_{g+\delta}^{\prime}$ and $y_{g+\delta}^{\prime}$, where $\delta=0,-1$ such that $w \in\left[x_{g}, y_{7}\right] \cap\left[x_{g+\delta}^{\prime}, y_{g+\delta}^{\prime}\right]$. Thus

$$
\Pi_{n+1}\left(A^{\prime} \times w\right) \subset\left[x_{g}, y_{g}\right] \cup\left[x_{g+\delta}^{\prime}, y_{g+\delta}\right],
$$

and condition (1) is satisfied.

THEOREM 3.5. For each component $A \subset A_{r}$ (where $A_{i}, i=1,2, \ldots$ are as defined above) and each $\varepsilon>0$ there exists an integer $N$ and a uniformly continuous homeomorphism $h: E^{n} \times E^{1} \rightarrow E^{n} \times E^{1}$ such that

1. $h=$ id in $E^{n+1}-A \times E^{1}$;

2. $\left|\Pi_{n+1}[h(x)]-\Pi_{n+1}(x)\right|<\varepsilon$;

3. For each $w \in E^{1}$ diameter of each component of $A_{N} \times w$ is less than $\varepsilon$.

Proof. Let $\varepsilon^{\prime}=\varepsilon / 8$ then by Lemma 3.4 there exists a uniformly continuous homeomorphism $h_{1}$ and an integer $N$ satisfying

a. $h_{1}=$ id on $E^{n+1}-A \times E^{1}$,

b. $\left|\Pi_{n+1} h_{1}(x)-\Pi_{n+1}(x)\right|<4 m+2$ for some positive integer $m$, and

c. diam $\Pi_{n}^{*}\left(A^{\prime} \times w\right)<4 \varepsilon^{\prime}$ for all $w \in E^{1}$ and components $A^{\prime}$ in. $A_{N} \cap A$.

Let $h_{2}: E^{n} \times E^{1} \rightarrow E^{n} \times E^{1}$ be the homeomorphism given by

$$
h_{2}(x, t)=\left(x, \frac{4 m+2}{\varepsilon^{\prime}} t\right) \text {. }
$$

The homeomorphism $h=h_{2}^{-1} h_{1} h_{2}$ is the desired homeomorphism.

Note that $h$ is isotopic to the identity since the homeomorphisms of Lemmas 3.3 and 3.4 were.

Theorem 3.6. $X_{n} \times E^{1}=E^{n+1}$ if $X_{n} \in \Gamma_{n}$, where $\Gamma_{n}$ is defined above.

Theorem follows from Theorem 2.1 and the following theorem which Is due to R. H. Bing [4].

THEOREM. Let $X_{n} \in \Gamma_{n}$. Further suppose that for each $i$ and $\varepsilon>0$ there is an integer $N$ and an isotopy $\mu$ of $E^{n+1}$ onto $E^{n+1}$ such that $\mu_{0}$ is the identity $\mu_{1}$ is uniformly continuous and

1. $\mu_{t}=\mathrm{id}$ on $E^{n+1}-\left(A_{j} \times E^{n}\right)$;
2. $\left|\Pi_{n+1} \mu_{t}(x)-\Pi_{n+1}(x)\right|<\varepsilon$, where $\Pi_{n+1}$ is the projection of $E^{n+1}$ onto the $(n+1)$-st coordinate;

3. For each $w \in E^{1}$ the diameter of each component of $\mu_{1}\left(A_{N} \times w\right)$ is less than $\varepsilon$.

Then $X_{n} \times E^{1}=E^{n+1}$.

Remark 3.4. Note that there exists a countable collection of compact sets $R_{i}$ such that
1. $A \times E^{1}=\bigcup_{i} R_{i}$,
2. $h\left(R_{i}\right) \subset R_{i}$ for all $i=1,2, \ldots$;
3. $h /$ Bdy $R_{i}=$ id for all $i=1,2, \ldots$,
4. $\operatorname{diam} \Pi_{n+1}\left(R_{i}\right)<\varepsilon / 8$, and
5. $\operatorname{diam} h\left(R_{i} \cap\left(A_{n} \times E^{1}\right)\right)<\varepsilon / 2$, where $h$ is the homeomorphism of Theorem 3.5.

4. The "Dogbone space" squared is $E^{6}$. In [7] K. W. Kwun showed that there exist two non-manifolds whose product is $E^{n}$ for $n \geqslant 6$. In this that there exist two non-manifolds whall show that the product of any two spaces belonging to $\Gamma_{n}$ and $\Gamma_{m}$ respectively is $E^{n+m}$.

Throughout this section let $\left\{A_{i}\right\}$ be as defined above and let $\left\{B_{i}\right\}$ be a collection of $m$-manifolds in $E^{m}$ which are analogous to the $A_{i}$. That is $B_{i}(i=1,2, \ldots)$ is a collection of compact manifolds in $E^{m}$ satisfying P1 and P2, where $B_{\infty}=\bigcap_{i} B_{i}$.

Lemira 4.1. Given $A$ and $B$ components of $A_{r}$ and $B_{s}$ respectively and $\varepsilon>0$ then there exists an integer $N>\max (r, s)$ and a homeomorphism $h: E^{n} \times E^{m} \rightarrow E^{n} \times E^{m}$ such that

1. $h=$ id on $E^{n+m}-(A \times B)$ and

2. Diam $h\left(A^{\prime} \times B^{\prime}\right)<\varepsilon$ for each component $A^{\prime} \subset A_{N} \cap A$ and $B^{\prime}$ $C B_{N} \cap B$.

Proof. By Lemma 3.1 there exist integer $J$ and $K$, a set of $n$-cells $E_{1}, \ldots, E_{p}$, and a set of $m$-cells $F_{1}, \ldots, F_{q}$ such that
1. $E_{i} \subset \operatorname{int} A$ for each $i=1,2, \ldots, p$;
2. $F_{j} \subset \operatorname{int} B$ for each $j=1,2, \ldots, q$;
3. For each component $A^{\prime} \subset A_{J} \cap A$ there is at least one $i$ such that $A^{\prime} \subset \operatorname{int} E_{i}$

4. For each component $B^{\prime} \subset B_{K} \cap B$ there is at least one $j$ such that $B^{\prime} \subset \operatorname{int} F_{j}$.

Let $N=\max (J, K)$ and note that for each component

$$
A^{\prime} \times B^{\prime} C\left(A_{N} \times B_{N}\right) \cap(A \times B)
$$

there exist integers $i$ and $j$ such that $A^{\prime} \times B^{\prime}$ is a subset of $\operatorname{int} E_{i} \times \operatorname{int} F_{j}$. 
By Lemma 2.2 there exists a collection of $(n+m)$ - cells $G_{1}, G_{2}, \ldots, G_{l}$ such that

1. For each component $A^{\prime} \times B^{\prime}$ of $\left(A_{N} \times B_{N}\right) \cap(A \times B)$ there exists .a unique $k$ such that $A^{\prime} \times B^{\prime} \subset \operatorname{int} G_{k}$ and $A^{\prime} \times B^{\prime} \cap G_{j}=\varnothing$ for all $j \neq k$.

2. $G_{k} \subset \operatorname{int} E_{i} \times \operatorname{int} F_{j} \subset A \times B$ for some $i$ and $j$.

Note that even though $i \neq j$ it may be the case that $G_{i} \cap G_{j} \neq \emptyset$. Nevertheless there exists an $(n+m)$-cell $Q_{i} \subset G_{i}$ whose diameter is less than $\varepsilon$ and such that $Q_{i} \cap G_{j}=\emptyset$ for $i \neq j$. For each component $A^{\prime} \times B^{\prime}$ $C\left\{\left(A_{N} \times B_{N}\right) \cap(A \times B)\right\}$ there exists an integer $i$ and a homeomorphism $h_{i}: E^{n+m} \rightarrow E^{n+m}$ such that
1. $A^{\prime} \times B^{\prime} \subset G_{i}$
2. $h_{i}=$ id on $E^{n+m}-G_{i}$,
3. $h_{i}\left(A^{\prime} \times B^{\prime}\right) \subset Q_{i}$.

Define $h=h_{1} \circ h_{2} \circ \ldots \circ h_{l}$. Even though the $G$ 's are not disjoint, $h_{i}$ is the identity on $G_{j} \cap\left(A_{N} \times B_{N}\right)$ for $j \neq i$. Thus $h$ satisfies conditions 1 and 2 of the theorem.

Remark 4.1. Since the homeomorphism $h$ of Lemma 4.1 is the identity outside a compact set $h$ is uniformly continuous and isotopic to the identity.

Theorem 4.2. Let $A_{i}, i=1,2, \ldots ; B_{j}, j=1,2, \ldots$ be defined as above, then there exists a pseido-isotopy $H: E^{n+m} \times I \rightarrow E^{n+m}$ such that

a. $H(x, 0)=x$;

b. If $H_{t}(x)=H(x, t)$ then for all $t<1, H_{t}$ is a homeomorphism of $E^{n+m}$ onto itself which is the identity outside a compact set;

c. $H_{1}$ maps $E^{n+m}$ onto itself and maps each component of $A_{\infty} \times B_{\infty}$ onto a distinct point

$$
\begin{aligned}
& \text { d. If } x \in E^{m+n}-\left(A_{\infty} \times B_{\infty}\right) \text { then } \\
& \qquad H_{1}^{-1}\left(H_{1}(x)\right)=x .
\end{aligned}
$$

Proof. Let $\varepsilon_{0}=\operatorname{diam}\left(A_{1} \times B_{1}\right)$ and $\varepsilon_{i}=\left(\frac{1}{2}\right)^{i}$ for $i=1,2, \ldots$ A sequence of integers $1=N(1), N(2), \ldots$ and isotopies,

for $i=1,2, \ldots$ which satisfy

$$
H^{i}: E^{n+m} \times\left[\frac{i-1}{i}, \frac{i}{i+1}\right] \rightarrow E^{n+m}
$$

$$
\begin{aligned}
& \text { 1. } H^{1}(x, 0)=x, \\
& \text { 2. } H^{i-1}\left(x, \frac{i-1}{i}\right)=H^{i}\left(x, \frac{i-1}{i}\right) \text { for } i=2,3, \ldots, \\
& \text { 3. diam } H^{i}\left(A^{\prime} \times B^{\prime}, \frac{i}{i+1}\right)<\varepsilon_{i} \text { for each component } A^{\prime} \times B^{\prime} \subset A_{N(i+1)} \times \\
& B_{N(i+1)},
\end{aligned}
$$

4. $H^{i}(x, t)=H^{i-1}\left(x, \frac{i-1}{i}\right)$ for $x \in E^{n+m}-\left(A_{N(i)} \times B_{N(i)}\right)$ and $i=2,3, \ldots$,
5. $\left\|H^{i}(x, t)-H^{i}\left(x, t^{\prime}\right)\right\|<\varepsilon_{i-1}$ for all $x \in E^{n+m}$ and $t, t^{\prime} \in\left[\frac{i-1}{i}, \frac{i}{i+1}\right]$ are defined inductively as follows. Let $A_{r}$ and $B_{s}$ of Lemma 4.1 be $A_{1}$ and $B_{1}$ respectively and let $\varepsilon$ of Lemma 4.1 be $\varepsilon_{1}$. Then there exists a uniformly continuous isotopy

$$
h_{1}: E^{n+m} \times I \rightarrow E^{n+m}
$$

and an integer $N(2)$ such that

$$
h_{1}(x, 0)=x,
$$

$\operatorname{diam} h_{1}\left(A^{\prime} \times B^{\prime}, 1\right)<\varepsilon_{1} \quad$ for each component,

$$
A^{\prime} \times B^{\prime} \subset A_{N(2)} \times B_{N(2)} \text { and } h_{1}(x, t)=x \text { on } E^{n+m}-\left(A_{1} \times B_{1}\right) \text {. }
$$

Define $H^{\prime}(x, t)=h_{1}(x, 2 t), 0 \leqslant t \leqslant \frac{1}{2}$.

suppose $B^{k}$ and $N_{k+1}$ are defined. Since $H_{w}^{k}$ is uniformly continuous for $w=\frac{k}{k+1}$ there exists a $\delta>0$ such that if the diameter of $V \subset E^{n+m}$ is less than $\delta$ then the diameter of $H_{w}^{k}(V)$ is less than $\varepsilon_{k+1}$. Lemma 4.1 implies the existence of an integer $N_{k+2}$ and an isotopy such that

$$
\begin{gathered}
h_{k+1}(x, 0)=x \text { on } E^{n+m}, \\
h_{k+1}(x, t)=x \text { on } E^{n+m}-\left(A_{N(k+1)} \times B_{N(k+1)}\right) \\
\operatorname{diam}\left(A^{*} \times B^{*}, 1\right)<\delta \text { for each component, } \\
A^{*} \times B^{*} \subset A_{N(k+2)} \times B_{N(k+2)} \text { and } h_{k+1} \text { is uniformly continuous. }
\end{gathered}
$$

Define

$$
H^{k+1}(x, t)=H_{w}^{k} h_{k+1}\left(x,(k+1)(k+2)\left(t-\frac{k}{k+1}\right)\right) \quad \text { for } \quad \frac{k}{k+1} \leqslant t \leqslant \frac{k+1}{k+2} .
$$

Clearly 1 and 2 are satisfied. Now

$$
H^{k+1}\left(x, \frac{k+1}{k+2}\right)=H_{w}^{k} h_{k+1}(x, 1)
$$

thus by choice of $\delta$ condition 3 is satisfied. Further $h_{k+i}(x, t)=x$ for thus by choice of $\delta$ condition 3 is satistied. Further $\left.h_{k+i}(x, t)^{\prime \prime} \times B^{\prime \prime}, t\right)$
$x \in E^{n+m}-\left(A_{N(k+1)} \times B_{N(k+1)}\right)$ hence condition 4 is satisfied. $h_{k+1}\left(A^{\prime \prime} \times B^{\prime}\right.$ is contained in $A^{\prime \prime} \times B^{\prime \prime}$ for each component $A^{\prime \prime} \times B^{\prime \prime} \subset A_{N(k+1)} \times B_{N(k+1)}$. $\operatorname{Diam}\left(H_{w}^{k}\left(A^{\prime \prime} \times B^{\prime \prime}\right)\right)<\varepsilon_{k}$ be condition 3 , thus condition 5 is satisfied. Define

$$
H(x, t)=H^{i}(x, t) \quad \text { on } \quad E^{n+m} \times\left[\frac{i+1}{i}, \frac{i}{i+1}\right] \quad \text { for } \quad i=1,2, \ldots
$$


and define $H(x, 1)=H_{1}(x)=\lim _{t \rightarrow 1} H(x, t) . H_{1}(x)$ is a continuous map of $E^{n+m}$ onto $E^{n+m}$ by condition 5. Clearly 1 implies that a is satisfied by $H$. Condition 4 along with definition of $H^{1}$ implies $\mathrm{b}$ is satisfied by $H$. Suppose $\varepsilon>0$ and $\alpha \times \beta$ is a component of $A_{\infty} \times B_{\infty}$ then there exists an integer $p$ such that $\left(\frac{1}{2}\right)^{P}=\varepsilon_{p}<\varepsilon$. For all $t>p /(p+1)$, $\operatorname{diam} H\left(A^{*} \times\right.$ $\left.\times B^{*}, t\right)<\varepsilon_{p}$ where $A^{*} \times B^{*}$ is the component of $A_{N(p)} \times B_{N(p)}$ containing $a \times \beta$. Thus $H(\alpha \times \beta, 1)$ is a point. Let $x \in E^{n+m}-A_{\infty} \times B_{\infty}$ then there exists an integer $N(q)$ such that $x \in \mathbb{E}^{n+m}-\left(A_{N(q)} \times B_{N(q)}\right)$. Thus 4 implies that $H(x, t)=H\left(x, \frac{q-1}{q}\right)$ for all $t>\frac{q-1}{q}$. But $H / E^{n+m} \times\left[0, \frac{q-1}{q}\right]$ is an isotopy thus $H_{1}^{-1}\left(H_{1}(x)\right)=x$ and $d$ is satisfied by $H$. Let $\alpha_{1} \times \beta_{1}$ and $\alpha_{2} \times \beta_{2}$ be distinct components of $A_{\infty} \times B_{\infty}$ then there exists an integer $N(j)$ such that $a_{1} \times \beta_{1} \subset A^{\prime} \times B^{\prime}$ and $a_{2} \times \beta_{2} \subset A^{\prime \prime} \times B^{\prime \prime}$, where $A^{\prime} \times B^{\prime}$ and $A^{\prime \prime} \times B^{\prime \prime}$ are distinct components of $A_{N(j)} \times B_{N(j)}$. Thus $H_{1}\left(a_{1} \times \beta_{1}\right)$ $\neq H_{1}\left(\alpha_{2} \times \beta_{2}\right)$ and $\mathrm{c}$ is satisfied. Therefore $H$ is the desired pseudo-isotopy.

CoRoLLARY 4.2. Suppose $F$ is an upper semi-continuous decomposition of $E^{n+m}$ consisting of the 2 -cetls $\alpha \times \beta$, where $\alpha \subset A_{\infty}$ and $\beta \subset B_{\infty}$ and the points of $E^{n+m}-\left(A_{\infty} \times B_{\infty}\right)$. If $Z$ is the decomposition space associated with $F$ then $Z$ is topologically $\mathbb{E}^{n+m}$. Moreover, there exists a uniformly continuous homeomorphism carrying $Z$ onto $E^{n+m}$.

THEOREM 4.3. Suppose $X_{n} \in \Gamma_{n}$ and $X_{m} \in \Gamma_{m}$ then $X_{n} \times X_{m}$ is topologically $E^{n+m}$.

Proof. By Corollary 4.2 there exists a pseudo-isotopy $H$ of $E^{n+m}$ onto itself which shrinks éach of the 2 -cells $\alpha \times \beta$ for $\alpha \subset A_{\infty}$ and $\beta \subset B_{\infty}$. Let $f=H_{1}$. The proof will be completed by constructing a pseudoisotopy $K$ of $f\left(E^{n+m}\right)$ onto itself which shrinks each of the arcs $f(\alpha \times y)$, $f(z \times \beta)$ where $\alpha$ is an are of $A_{\infty}, \beta$ is an arc of $B_{\infty}, z \in E^{n}$ and $y \in E^{m}$. Let

and

$$
U_{1}=\bigcup_{i} f\left(\operatorname{int} A_{i} \times\left(E^{m}-B_{i}\right)\right)
$$

$$
U_{2}=\bigcup_{i} f\left(\left(E^{n}-A_{i}\right) \times \operatorname{int} B_{i}\right) .
$$

Note that each arc $f(\alpha \times y) \subset U_{1}$ and $f(z \times \beta) \subset U_{2}$. Also $U_{1} \cap U_{2}=\varnothing$.

The pseudo-isotopy $K$ can be constructed by amending the construction of the pseudo-isotopy in [7] as follows.

(1) Replace the compact neighborhoods $T_{i}$ and $T_{i}^{\prime}$ with $A_{i}$ and $B_{i}$ respectively.

(2) In the proof of the Lemma replace Theorem 1 of [1] with Theorem 3.6 of this paper. And further replace the $R_{i}^{\prime}$ by $R_{i}$ of Remark 3.4
[1] J. J. Andrews and M. L. Curtis, n-space modulo an arc, Ann. of Math. 75 (1962), pp. 1-7.

[2] S. Armentrout, Monotone decompositions of $E^{3}$ in Topology Seminar, Wisconsin 1965, 1966, pp. 1-25.

[3] R. H. Bing, A decomposition of $E^{3}$ into points and tame ares such that the decomposition space is topologically different from $E^{3}$, Ann. of Math. 65 (1957), pp. $484-500$.

[4] - The cartesian product of a certain non-manifold and a line is $E^{4}$, Ann. of Math. 70 (1959), pp. 399-412.

[5] - Decompositions of $E^{3}$ in Topology of 3-Manifolds and Related Topics, 1962, pp. 5-21.

[6] J. G. Hocking and G. S. Young, Topology, 1961

[7] K. W. Kwun, Product of Euclidean spaces modulo an are, Ann. of Math. 79 (1964), pp. 104-107.

INSTITUTE FOR ADVANCED STUDY Princeton, New Jersey

Reçu par la Rédaction le 3.4. 1969 